\title{
UMA REVISÃO DE REVISÓES POR OCASIÃO DOS 70 ANOS DE O COMPORTAMENTO DOS ORGANISMOS DE B. F. SKINNER
}

\section{A REVIEW OF REVIEWS ON THE 7OTH ANNIVERSARY OF B.F. SKINNER'S THE BEHAVIOR OF ORGANISMS}

\author{
João Claudio Todorov
}

UNIVERSIDADE CATÓLICA DE GOIÁS E INSTITUTO DE EDUCAÇÃO SUPERIOR DE BRASÍLIA, BRASIL

\begin{abstract}
RESUMO
Ao longo de 70 anos o livro O Comportamento dos Organismos de Skinner (1938) provocou profundo impacto na psicologia, aparecendo ao mundo como um sistema para a organização de dados experimentais e de observação e a descrição de um programa de pesquisa totalmente inovador. Propôs a criação da teoria pela indução a partir dos dados observados para então prosseguir com o levantamento de hipóteses e seu teste empírico, contrariando toda a zeitgeist hipotético-dedutiva da época. O presente trabalho comemora esse septuagésimo aniversário revisando as revisōes críticas que o livro recebeu de autores importantes como Ernest R. Hilgard, Philip N. Hineline, J. W. Donahoe e D. C. Palmer.
\end{abstract}

Palavras-chave: Skinner, análise experimental do comportamento, programa de pesquisa, organização de dados experimentais.

\section{ABSTRACT}

B. F. Skinner's The Behavior of Organisms (Skinner, 1938) made a lasting impact in the world of psychology as a system for the organization of experimental data and data from formal and informal observations, and as the description of a totally innovative research program. In contrast to the hypothetico-deductive Zeitgeist of the time, Skinner offered a way to theorize through induction, beginning with observed data that lead to hypotheses which are then tested. The present paper celebrates this anniversary considering reviews of the book that have been published by important authors like Ernest R. Hilgard, Philip N. Hineline, J. W. Donahoe, and D. C. Palmer.

Keywords: Skinner, experimental analysis of behavior, research programme, organization of experimental data.

Quando "O Comportamento dos Organismos” de Skinner (1938) foi publicado, Ernst R. Hilgard, um professor da Stanford University, já era um nome consagrado da psicologia americana. Um artigo seu sobre o livro, publicado no "Psychological Bulletin" da American Psychological Association, mostra a importância que o meio acadêmico atribuiu à obra. Skinner era conhecido desde sua dissertação de doutorado sobre o conceito de reflexo. "Skinner propõe um sistema para uma formulação conveniente de dados comportamentais, e então descreve experimentos que testam o sistema.": assim simplesmente Hilgard resume o livro (Hilgard, 1939, p. 121), e formula três perguntas cujas respostas poderiam ser uma medida do sucesso ou fracasso do autor na busca de seus objetivos. As perguntas são tão pertinentes que ainda hoje servem para que se avalie o estado da análise do comportamento como um todo:

“(1) - Quais são as principais características do sistema que ele propõe?

(2) - Que significância têm os achados experimentais, tanto quanto validação do sistema quanto em relação às questôes da psicologia formuladas de outras maneiras?

(3) - O sistema como formulado e apoiado experimentalmente tem alguma probabilidade

O autor é bolsista de produtividade do CNPq. Correspondência para o e-mail: joaoclaudio.todorov@gmail.com. 


\section{J. C. TODOROV}

de vir a competir com outros sistemas da psicologia?” (Hilgard, 1939, p. 121).”

Para Hilgard, a primeira marca distintiva do sistema proposto por Skinner é seu caráter de ciência estritamente do comportamento, nunca uma ciência da mente ou uma ciência do sistema nervoso, acompanhando nesse ponto vários teóricos importantes da época, como Guthrie, Hull, Lewin e Tolman, mas diferindo profundamente quanto ao modo de trabalhar: para Skinner a dedução e teste de hipóteses são atividades subsidiárias em uma ciência descritiva, que chega a leis por indução baseada no comportamento observável. Essa marca distintiva é ao mesmo tempo seu ponto forte e seu calcanhar de Aquiles, pois como Skinner bem sabia, é totalmente contrária à maneira de pensar da cultura cristã ocidental, que faz do teste de hipóteses o carro chefe da ciência (Hilgard, 1939, p. 283). Quanto à natureza desse sistema, Hilgard ressalta que a estrutura de um sistema descritivo é determinada pela natureza de seu objeto de estudo. Ao escolher o reflexo como unidade de análise, uma unidade redefinida sem conotaçôes neurais, Skinner reescreve as leis do reflexo como formuladas por Sherrington (1906/1961), Magnus (1924) e Pavlov (1927), em termos puramente comportamentais. Contudo, quando essas "leis" deixam de se referir explicitamente a reflexos espinhais, elas passam a ser hipóteses a serem estudadas, e o programa de pesquisa de Skinner se dirige para o teste de hipóteses levantadas por sua redefinição do reflexo como correlação entre estímulos e respostas:

"As stated, they do not appear to the reviewer to be laws at all, but collections of variables probably correlated in such ways that laws might be looked for." (Hilgard, 1939, p. 122).

As "leis" com as quais Skinner iniciou seu programa de pesquisa eram, para Hilgard, mais hipóteses de trabalho que orientariam experimentos. Mas a crença de Skinner em um programa de construção de teoria via indução baseada em dados de observação é recompensada pela formulação e reconhecimento de dois tipos de aprendizagem, operante e respondente, nomes novos para comportamentos conhecidos mas até então tratados como um mesmo tipo (Hilgard, 1939, p. 122). Neste ponto, Staddon (2001) apenas repete Hilgard: não há nada de realmente novo na distinção operante/respondente, algo implícito já em Thorndike (1911).

As leis do reflexo, que ocupam muitas páginas de "O Comportamento dos Organismos" e deveriam ser a base de uma teoria construída "de baixo para cima", do reflexo ao pensamento, ainda receberam muita atenção em "Princípios de Psicologia" de Fred S. Keller e W. N. Schoenfeld (1950/1968), mas ocupam cada vez menos espaço em livros didáticos, como Millenson (1967/1975), Catania (1984), e Donahoe e Palmer (1994). A análise do comportamento se especializa em comportamento operante, tratado como se fosse independente de sua base reflexa. Uma exceção recente é o trabalho de Travis Thompson (2007); outra, mais antiga, é o trabalho de Jack Michael (1982) sobre operações estabelecedoras, mas a falta de repercussão desse trabalho é indicativa de seu pequeno impacto.

Para Hilgard, essa preferência por operantes em detrimento de respondentes vem do fato de Skinner desenvolver seu trabalho inicial como uma quebra de uma ligação com as neurociências, aí incluindo Pavlov (1927), sem preocupação com os outros sistemas em desenvolvimento na psicologia dos anos 30 do século passado:

\footnotetext{
"Embora seja eloqüente ao denunciar uma ciência do comportamento que se subordina às
} 
neurociências, ele está mais obviamente cônscio dos neurologistas e fisiologistas do que dos psicólogos. O comportamento respondente não é proeminente nos trabalhos de Ebbinghaus, Freud, McDougal, na fase mais tardia de Thorndike, na Gestalt, e em muitos outros sistemas menos fisiológicos que o de Skinner”. (Hilgard, 1939, p. 123).

Skinner não só não discute seu sistema em relação aos principais sistemas da psicologia da época, ele também mal discute os sistemas mais diretamente ligados ao seu, os competidores diretos em relação às teorias da aprendizagem, Lewin (1936), Tolman (1932), Hull (1943) e Guthrie (1935). Para um livro novo, de autor novo, a falta dessa discussão dificultou muito sua popularização. Como disse Hilgard: "Se Skinner não foi capaz de relacionar sua obra aos trabalhos de outros pesquisadores, como se pode exigir de um leitor, que desconhece seu trabalho, que faça por si só essas transições? (Hilgard, 1939, p. 124). Esses inconvenientes, um apego excessivo às neurociências de um lado (ainda quando as critica) e um distanciamento da psicologia como um todo, prejudicaram o livro, para Hilgard (1939), e dificultaram sua divulgação, levando a um impacto bem menor do que merecia. Hilgard ainda sugere, como Tolman o faria independentemente, que se Skinner tivesse inserido em sua caixa experimental uma segunda barra, possibilitando o estudo de situações de escolha, seu trabalho ficaria melhor, mais próximo de Hull (1943).

Como viria a acontecer em várias outras situações, Skinner não seguiu as sugestões de imediato e nunca as citou, assim como nunca citou diretamente seus críticos. A questão do estudo experimental do comportamento de escolha só é retomada em "Are theories of learning necessary?" (Skinner, 1950). A extensão para áreas abertas por outros sistemas é vagarosa, meticulosa em vários pontos que se referem ao comportamento de indivíduos, como em "The operational analysis of psychological terms" (Skinner, 1945) e "O Comportamento Verbal" (Skinner, 1957), e apenas sugestiva na maior parte do que se refere ao comportamento em grupos, organizações e sociedades (Skinner, 1953/1967). Para quem no início da carreira não se preocupava com o que pensava o "establishment" da psicologia, Skinner, depois de famoso, vai se dedicar principalmente aos que o criticavam politicamente, sem mencionar seus críticos (comportamento típico de políticos, mas não de intelectuais), em "About Behaviorism" (1974), 'Beyond Freedom and Dignity" (1971), e "Contingencies of Reinforcement" (1969), entre outros.

A crítica de Hilgard é positiva, afinal, ao chamar atenção para as novas descobertas e o enorme potencial representado pelos esquemas de reforço intermitente. Suas críticas são importantes, e teriam ajudado a análise do comportamento se levadas em conta a tempo. Não foram, nem por Skinner, nem pelos seguidores de então (à exceção talvez de F. S. Keller e W. N. Schoenfeld em "Princípios de Psicologia" (Keller \& Schoenfeld, 1950/1968). Resumindo, as sugestões de Hilgard poderiam ser aproveitadas por um autor interessado em produzir um livro didático mais completo da maneira que segue:

1. Skinner não inventa dois tipos de condicionamento nem dois tipos de comportamento (Staddon, 2001), mas tem o mérito de abandonar a busca de uma linguagem pavloviana que unifique os dois tipos em um único sistema explicativo, como vinham fazendo, por exemplo, Hull (1943) e Guthrie (1935).

2. Começar pelos reflexos é respeitar a história e desconhecer os interesses do leitor. 


\section{J. C. TODOROV}

Se a importância do capítulo 1 só aparece depois do capítulo 10, haja controle sobre o comportamento do leitor para fazê-lo chegar até lá. Deve-se deixar claro que o livro abordará tudo o que o leitor entende por psicologia e muito mais, pois análise do comportamento é um maneira de se fazer psicologia, não apenas uma parte (talvez a menos interessante) dela.

3. Depois e por causa de "O Comportamento dos Organismos" e de Skinner, praticamente não há quem na psicologia trabalhe sem usar comportamento como variável dependente (Roediger, 2004).

\section{Hineline}

Hineline analisa "O Comportamento dos Organismos" cinqüenta anos depois de Hilgard, com a vantagem de não ter que prever, só explicar o que aconteceu com a análise do comportamento. Em termos gerais, concorda com a crítica de Hilgard, mas vai mais longe, escreve um trabalho mais pormenorizado, mostrando, por exemplo, como a linguagem reflexológica predomina nos primeiros capítulos, mesmo quando Skinner fala de comportamento operante (Hineline, 1990, p. 316). A linguagem moderna da análise operante do comportamento só aparece a partir da página 241. Daí em diante Skinner ${ }^{1}$ realmente introduz uma novidade para a psicologia experimental americana de seu tempo: uma teoria com interpretaçôes baseadas no ambiente, no lugar de teorias baseadas no que acontece no organismo (Hineline, 1990, p. 317).

O desenvolvimento posterior do trabalho de Skinner veio a ser caracterizado por essa interpretação baseada no ambiente, e muitos analistas do comportamento hoje afirmam que se não for assim não é análise do comportamento. Mas em "O Comportamento dos Organismos" Skinner recai em interpretaçôes baseadas no organismo sempre que tem que falar em comportamentos complexos que envolvem interações operante-respondente, como no caso das emoções (Skinner, 1938, p. 341). Em que pese a declaração de Skinner de que sempre tratou de assuntos como condicionamento, motivação e emoção como diferentes conjuntos de variáveis das quais a probabilidade de resposta é função, não há na análise do comportamento um trabalho sistemático envolvendo os dois últimos "conjuntos de variáveis" como há para condicionamento.

Para Hineline, o que fica até hoje como herança de "O Comportamento dos Organismos" é o uso da taxa de respostas como medida do comportamento (e por conseqüência a inclusão do tempo como variável), contribuição original inequívoca de Skinner, a consistência lógica da teoria e a rejeição de termos mentalistas. ${ }^{2}$ Como conseqüência do uso da taxa de respostas como variável dependente Hineline vê três implicações do trabalho desenvolvido no livro:

(a) A análise do comportamento passa a ser o estudo, definição e caracterização de ambientes efetivos em processos de interação ambiente-comportamento.

(b) Um processo psicológico é compreendido como uma interação ambiente-comportamento.

(c) Taxa de respostas é uma abstração, ainda que composta de eventos tangíveis,

\footnotetext{
${ }^{1}$ A dissertação de doutorado de Skinner, defendida em 1931, serviu de base para o livro. A dissertação foi escrita antes da distinção operante-respondente e usa apenas termos e conceitos pavlovianos, o que talvez explique a linguagem desigual apontada por Hineline.

${ }^{2}$ A difusão da análise do comportamento, entretanto, só veio a ocorrer depois de "Princípios de Psicologia" (Keller \& Schoenfeld, 1950/1968) e das mudanças no ensino de psicologia experimental na Universidade de Columbia, em Nova Iorque, introduzidas por Fred S. Keller e W. N. Schoenfeld.
} 
observáveis, uma abstração difícil de ser entendida pelo leigo, da mesma maneira que foi difícil para muitos psicólogos entender a redefinição de Skinner para reflexo, outra abstração, uma relação entre classes de eventos observáveis, e nada mais.

Neste ponto é interessante notar como a análise do comportamento realmente se desenvolveu mais na linha apontada por Hineline em "O Comportamento dos Organismos" do que no trabalho mais completo de "Ciência e Comportamento Humano", no qual Skinner defende e desenvolve trabalhos de observação, de análise funcional e de análise conceitual, muito além de apenas trabalhos de análise experimental do comportamento.

\section{Donahoe e Palmer}

Um dos tópicos mais bem discutidos por Skinner em "O Comportamento dos Organismos" é o do uso do conceito de inibição como explicação na psicologia. Segundo Staddon (1969) inibição é um dos termos mais antigos da psicologia, usado primeiramente por Weber e Weber (1845) na biologia e por Sechenov (1863) na psicologia. Para Skinner (1938) para ser útil, inibição deveria ser diretamente observada e medida, como na fisiologia. Nos neurônios alguns neurotransmissores são chamados de excitatórios porque produzem um potencial de ação; outros são chamados de inibitórios, porque dificultam a ocorrência de um potencial de ação. São diretamente observáveis e medidos. Como empregada na psicologia, inibição é apenas inferida do comportamento observado, e não pode ser aceita como explicação desse comportamento. Com essa análise Skinner praticamente mata o conceito de inibição na análise do comportamento e nunca o usa como explicação. Donahoe e Palmer (1988) revêem toda a discussão feita por Skinner e acompanham a ressurreição do conceito no Journal of the Experimental Analysis of Behavior entre 1968 e 1975, quando foram publicados 61 artigos com a palavra-chave "inibição". Nos oito anos seguintes foram publicados apenas 13 artigos, e de lá para cá o assunto praticamente desaparece. Mesmo de 1968 a 1975, inibição surge não como parte do programa de pesquisa (Lakatos, 1978) da análise do comportamento, mas como uma resposta a eventos ocorrendo em outro programa, da psicologia experimental cognitiva (Rescorla, 1969), ainda que o procedimento experimental utilizado seja típico da análise experimental do comportamento (Guttman \& Kalish, 1956; Honig, Boneau, Burstein \& Pennypacker, 1963; Jenkins \& Harrison, 1962; Brown \& Jenkins, 1967). A ausência de trabalhos oriundos do programa original de Skinner (1938) é mais estranho quando se recorda, como o fazem Donahoe e Palmer (1988), que Skinner dedicou muitas páginas para imaginar possíveis explicações para situações que vinham sendo explicadas com o conceito de inibição. Donahoe e Palmer concluem que o conceito desapareceu da análise do comportamento simplesmente porque não tem função, nem científica, nem heuristicamente. Mais uma vez, os dados são produzidos fora da análise do comportamento: quem comprova a tese de Skinner de 1938 são Rescorla e Wagner (1972), que mostraram que a inibição condicionada pode ser tratada como um caso especial de discriminação de estímulos.

Dado o exposto, cabem mais alguns conselhos ao autor de livro didático acima mencionado: -Lembre-se que muito do que interessa à análise do comportamento ocorre fora dela. Mire-se no exemplo de Keller e Schoenfeld 


\section{J. C. TODOROV}

(1950/1968) e não se prenda apenas no que é publicado no JEAB e no JABA. 'O Comportamento dos Organismos' completou 70 anos, Skinner morreu, a vida continua e a análise do comportamento está cada vez mais viva e atuante. Um livro de introdução não tem que seguir a estrutura nem de 'Princípios de Psicologia' (Keller \& Schoenfeld, 1950/1968), nem de 'Ciência e Comportamento Humano' (Skinner, 1953/1967).”

\section{REFERÊNCIAS}

Brown, P. L., \& Jenkins, H. M. (1967). Conditioned inhibition and excitation in operant discrimination learning. Journal of Experimental Psychology, 75, 255266.

Catania, A. C. (1984). Learning (2nd edition). Englewood Cliffs, NJ: Prentice-Hall.

Donahoe, J. W., \& Palmer, D. C. (1994). Learning and complex behavior. Boston, MA: Allyn and Bacon.

Donahoe, J. W., \& Palmer, D. C. (1988). Inhibition: a cautionary tale. Journal of the Experimental Analysis of Behavior 50, 333-341.

Guthrie, E. R. (1935). The psychology oflearning. New York, NY: Harper \& Row.

Guttman, N., \& Kalish, H. I. (1956). Discriminability and stimulus generalization. Journal of Experimental Psychology, 29, 390-400.

Hilgard, E. R. (1939) Review of B. F. Skinner's The Behavior of Organisms. Psychological Bulletin, 36, 121-125.

Hineline, P.N. (1990). The origins of environment-based psychological theory. Journal of the Experimental Analysis of Behavior, 53, 305-320.

Honig, W. K., Boneau, C. A., Burstein, K. R., \& Pennypacker, H. S. (1963). Positive and negative generalization gradients obtained after equivalent training conditions. Journal of Comparative and Physiological Psychology, 56, 111-116.
Hull, C. L. (1943). Principles of behavior. New York, NY: Appleton-Century-Crofts.

Jenkins, H. M., \& Harrison, R. H. (1962). Generalization gradients of inhibition following auditory discrimination learning. Journal of the Experimental Analysis of Behavior, 5, 435-441.

Keller, F. S. \& Schoenfeld, W. N. (1950/1968). Princípios de Psicologia (C. M. Bori, R. Azzi, trads.). São Paulo: EPU.

Lakatos, I. (1978). The methodology of scientific research programmes. Cambridge: Cambridge University Press. Lewin, K. (1936) Principles of topological psychology. New York, NY: McGraw-Hill

Magnus, R. (1924). Körperstellung. Berlin, 1924.

Michael, J. (1982). Distinguishing between discriminative and motivational functions of stimuli. Journal of the Experimental Analysis of Behavior, 37, 149-155.

Millenson, J. R. (1967/1975). Princípios de análise do comportamento. Brasília: Coordenada.

Pavlov, I. P. (1927). Conditioned reflexes (G. V. Anrep, Trans.). Londres: Oxford University Press.

Rescorla, (1969). Pavlovian conditioned inhibition. Psychological Bulletin, 72, 77-94.

Rescorla, R. A., \& Wagner, A. R. (1972). A theory of Pavlovian conditioning: Variations in the effectiveness of reinforcement and nonreinforcement. In A. H. Black, \& W. F. Prokasy (Orgs.), Classical conditioning II: Current research and theory (pp. 64-99). New York, NY: AppletonCentury-Crofts.

Roediger, H. L. (2004). What happened to behaviorism? American Psychological Society Observer, 17, 3.

Sechenov, I. M. (1863). Refleksy go lovnogo mozga. Traduzido para o inglês como "Reflexes of the brain" por A. A. Subkov em I. M. Sechenov, Selected works, Moscow and Leningrad state Publishing House for Biological and Medical Literature, 1935.

Sherrington, C. S. (1906/1961). The integrative action of the nervous system. New Haven, CT: Yale 
University Press.

Skinner, B. F. (1938). The behavior of organisms. New York, NY: Appleton-Century.

Skinner, B. F. (1945). The operational analysis of psychological terms. Psychological Review, 52, 270-277.

Skinner, B. F. (1950). Are theories of learning necessary? Psychological Review, 57, 193-216.

Skinner, B. F. (1953/1967). Ciência e comportamento humano. New York, NY: Macmillan.

Skinner, B. F. (1957). Verbal behavior. New York, NY: Appleton-Century-Crofts.

Skinner, B. F. (1969). Contingencies of reinforcement: $A$ theoretical analysis. New York, NY: AppletonCentury-Crofts.

Skinner, B. F. (1971). Beyond freedom and dignity. New York, NY: Knopf.

Skinner, B. F. (1974). About behaviorism. New York, NY: Knopf.
Staddon, J. E. R. (1969). Inhibition and the operant. Journal of the Experimental Analysis of Behavior, 12, 481-487.

Staddon, J. E. R. (2001). The new behaviorism: Mind, mechanism and society. Philadelphia, PA: Psychology Press.

Thompson, T. (2007). Relations among functional systems in behavior analysis. Journal of the Experimental Analysis of Behavior, 87, 423-440.

Thorndike, E. L. (1911). Animal intelligence. New York: McMillan.

Tolman, E. C. (1932). Purposive behavior in animals and men. New York, NY: Appleton-Century-Crofts.

Weber, E. F. W., \& Weber, E. H. (1845). Experimenta physiologica in theatro anatomico. Annali Universali di Medicina, Milão, 116, 225-233.

Recebido em 14 de maio de 2008 Aceito em 18 de dezembro de 2008 